\title{
SPEcial Section Guest EdITORIAL
}

Optical techniques provide effective means for the nondestructive investigation of different biological objects and tissues in general, and of biological fluids in particular. In comparison to other biological fluids, blood is attracting much more attention from researchers due to its fundamental and integrating role in the human organism. The knowledge of the optical properties of human blood plays an important role for many diagnostic and therapeutic applications in laser medicine and medical diagnostics. In many cases they can be related to structural, mechanical, and other parameters that strongly depend on the physiological status of the organism. This makes the more detailed study of the optical properties of blood and the development of novel techniques for blood diagnostics a real challenge.

Six papers are selected for this special section. In the first two articles the optical properties of blood are studied. In the article by A. Roggan et al., the integrating sphere technique and Monte Carlo simulations are applied to measure the scattering and absorption coefficients and the anisotropy factor of circulating blood at different levels of hematocrit, oxygen saturation, and osmolarity in the wavelength range from 400 to $2500 \mathrm{~nm}$. In the article by A. N. Yaroslavsky et al., the impact of the scattering phase function approximation on the optical properties of whole human blood determined from integrating sphere measurements is investigated. The presented data prove that the variations of the employed approximation (Mie, Gegenbauer kernel, or HenyeyGreenstein) can cause large discrepancies in the derived values of some optical parameters.

The third paper by V. L. Voeikov, C. N. Novikov, and N. D. Vilenskaya deals with the lucigenin- and luminol-dependent chemiluminescence in the samples of nondiluted human blood. The authors show that besides being a measure of oxidative metabolism in blood, the chemiluminescence data from measurements in nondiluted blood can be informative of integrative properties of blood tissue.
In the article by Y. Aizu and T. Asakura, the peculiarities of the retinal blood flow diagnostics are analyzed from the methodological point of view. Prior to this analysis a comparative review of the Dopplerand the speckle-based measurement techniques and the spectral reflectance properties of the ocular fundus tissue layers are given.

In the fifth article by A. V. Priezzhev et al. the possibilities of optical diagnostics of whole blood samples in vitro are discussed in relation to the measurement of the aggregational properties of red blood cells. The technique is based on the measurement of variations of the intensity of light backscattered from a thick layer of blood in stasis and at variable shear stress. The presented results show that it is possible to detect different changes of aggregational ability of red blood cells and to obtain reliable and reproducible data distinguishing normal blood samples and those with hemorheological disorders that can be related to different pathologies of the donors.

Optical diagnostics of individual red blood cells based on their deformation by high frequency electric field is discussed in the sixth paper V. L. Kononenko and T. A. Ilyina. Optically registered elongation of cells enables the determination and monitoring of their elastic moduli and viscous properties. However, an unambiguous evaluation of mechanical properties from the optical data is a complicated problem. The present work is aimed at the development of a comprehensive and experimentally verified theoretical basis for the dielectro-deformational investigations of red blood cells.

More articles on optical diagnostics of other biological fluids and model particle suspensions will be published in the future issues of the journal.

\section{Alexander V. Priezzhev Toshimitsu Asakura Special Section Guest Editors}

